Research Paper

\title{
Molecular variations in Vibrio alginolyticus and $V$. harveyi in shrimp-farming systems upon stress
}

\author{
Anix Vivek Santhyia, Rosalind George Mulloorpeedikayil, \\ Riji John Kollanoor, Prince M.J. Jeyaseelan \\ Department of Fish Pathology and Health Management, Tamilnadu Fisheries University, Tuticorin, India.
}

Submitted: May 14, 2014; Approved: December 28, 2014.

\begin{abstract}
A study was performed to investigate the genomic variations in the shrimp farm isolates of Vibrio alginolyticus and $V$. harveyi when the isolates were subjected to environmental stress. Samples of shrimps, water and sediment were collected from Southern Indian coastal shrimp farms. Vibrio isolates were biochemically identified and confirmed using $16 \mathrm{~S}$ rDNA and gyrB gene specific PCR. The bacterial strains were genotyped by PCR fingerprinting using GTG(5) and IS (Insertion Sequence) primers. Seven strains each of $V$. alginolyticus and $V$. harveyi were subjected to 10 passages through trypticase soya broth (TSB), which contained different $\mathrm{NaCl}$ concentrations (3, 6 and 8\%) and trypticase soya agar (TSA). V. alginolyticus was also passaged through TSB with a $12 \% \mathrm{NaCl}$ concentration. PCR fingerprinting, which was performed on the strains that were passaged through different salt concentrations, confirmed that $V$. alginolyticus and $V$. harveyi could affect the genomic variations, depending on the environmental conditions of the culture. The study highlights the complex genotypic variations that occur in Vibrio strains of tropical aquatic environment because of varied environmental conditions, which result in genetic divergence and/or probable convergence. Such genetic divergence and/or convergence can lead to the organismal adaptive variation, which results in their ability to cause a productive infection in aquatic organisms or generation of new strains.
\end{abstract}

Key words: Vibrio harveyi, Vibrio alginolyticus, molecular variation, shrimp, Penaeus monodon.

\section{Introduction}

Luminescent $V$. harveyi has been reported to cause serious infections in shrimp farming systems and can lead to a $100 \%$ mortality rate and, consequently, huge economic losses (Lavilla-Pitogo et al., 1990; Lavilla-Pitogo and de la Pena, 1998; Leano et al., 1998). Harveyi clade is now recognized as one of the 14 clades in the Vibrio genus (Sawabe et al., 2007), which consists of $V$. harveyi, $V$. campbellii, $V$. rotiferianus, $V$. parahaemolyticus, $V$. alginolyticus, $V$. natriegens and $V$. mytili, all of which share a high level of phenotypic and genotypic homology (Cano-Gomez et al., 2009). There are both virulent and avirulent strains in Vibrio harveyi; luminescent strains are virulent in almost all cases, although exceptions have been reported (Defoirdt et al., 2007). The expression of bioluminescence in $V$. harveyi is co-regulated with the production of toxin-A; hence, this bioluminescence expression is considered a virulence factor (Manefield et al., 2000). However, different factors, such as adhesion factors, extracellular polysaccharides and biofilm formation, lytic enzymes, siderophores, type III secretion systems and bacteriophages, appear to induce the virulence in $V$. harveyi (Ruwandeepika et al., 2012)

Pathogenicity and genomic variations have been found among the $V$. harveyi and $V$. alginolyticus isolates that were obtained from shrimp-farming systems (Hernandez and Olmos, 2004; George et al., 2005; Satendrakumar et al., 2007). Several processes such as horizontal gene transfer, prophage integration, super integrons, generation of pathogenicity islands via integration of plasmids, phages, or conjugative transposons into specific target genes 
have been suggested to generate pathogenic vibrios from the environmental isolates (Thompson et al., 2004).

Vibrios are dominant in the ocean and shrimp-farming environment because of their versatility in metabolic activity and their ability to serve as nitrifiers, as demanded by the environmental parameters (Grimes et al., 1984; Thangarani, 2001; Urakawa and Rivera, 2006). Starvation studies have indicated that $V$. alginolyticus and $V$. parahaemolyticus can have phenotypic alterations as a survival strategy (Abdallah et al., 2009). It was also observed that the changed osmolarity of the culture medium could change the outer membrane protein patterns of both $V$. alginolyticus (Xu et al., 2005) and $V$. parahaemolyticus (Xu et al., 2004). Therefore, the current study was performed to investigate whether genomic variability would develop in two important Vibrio strains of the shrimp farming systems viz. $V$. alginolyticus and $V$. harveyi under the effect of changed environmental culture conditions.

\section{Materials and Methods}

Samples of farmed shrimp, brood stock, larvae, gut and intestine of loose-shell-affected shrimps, shrimp farm water and sediment and hatchery water were collected from two Southeast Indian States, Tamilnadu and Andhra Pradesh, brought to the laboratory in ice and processed within 12-24 h. The shrimp samples were tested for the presence of white spot syndrome virus (WSSV) using diagnostic PCR with the standard procedures (Lo et al., 1996).

Seven strains of each $V$. alginolyticus and $V$. harveyi were isolated and biochemically identified (George et al., 2005; Satendrakumar et al., 2007) from the collected samples and were confirmed using gyrB gene specific PCR (Thaithongnum et al., 2006) and 16SrDNA analysis (Oakey et al., 2003) (Table 1). To study the genomic variations of the species under different culture environmental conditions, young cultures of the isolates, which were grown in trypticase soya broth, were further inoculated into a battery of culture tubes with media that contained various salt concentrations. All isolates were inoculated into a normal maintenance medium (TSA) with $1 \%$ salt concentra- tion. In addition, the $V$. harveyi strains were inoculated into three sets of sterile trypticase soya broth (TSB) (Hi Media, Mumbai, India) at 3, 6 and $8 \%$ sodium chloride $\mathrm{w} / \mathrm{v}$, whereas the $V$. alginolyticus strains were inoculated into four sets of $3,6,8$ and $12 \%$ salt concentrations. Each of these cultures was subcultured at a 24-h interval to another sterile culture medium of the identical composition. Then, each isolate was passaged for 10 days, and the DNA of the isolates was extracted at the initial 0 passage and at the end of 10 passages. For the DNA extraction, the isolates at 0 passage and at the end of 10 passages were grown in a sodium-chloride-supplemented Luria-Bertani (LB) broth $(1 \%$ tryptone, $0.5 \%$ yeast extract, $1.0 \% \mathrm{NaCl}, \mathrm{pH} 7.5)$ at $32{ }^{\circ} \mathrm{C}$ for $16-20 \mathrm{~h}$, and the cells were harvested and washed twice in physiological saline. The chromosomal DNA extraction was performed using standard phenol-chloroform and ethanol precipitation (Sambrook et al., 1989). The extracted DNA was dissolved in sterile deionized water (Biocel, Millipore, Molsheim, France) and used for the PCR analysis. PCR fingerprinting of the isolates were performed using IS-PCR (George et al., 2005) and GTG (5) PCR (Gomez-Gil et al., 2004).

For IS-PCR, amplification reactions were performed in $50 \mu \mathrm{L}$ with 1.5 unit of Taq polymerase, $20 \mathrm{mM}$ Tris $(\mathrm{pH}$ 8.3), $50 \mathrm{mM} \mathrm{KCl}, 1.5 \mathrm{mM} \mathrm{MgCl}_{2}$, and $200 \mu \mathrm{m} \mathrm{dNTP}$ (Genei, Bangalore, India). The reaction mixture was incubated at $94{ }^{\circ} \mathrm{C}$ for $2 \mathrm{~min}$, followed by 35 cycles at $94{ }^{\circ} \mathrm{C}$ for $45 \mathrm{~s}, 58^{\circ} \mathrm{C}$ for $45 \mathrm{~s}$ and $72{ }^{\circ} \mathrm{C}$ for 1 min with a final extension at $72{ }^{\circ} \mathrm{C}$ for $20 \mathrm{~min}$ in a Mastercycler (Eppendorf, Hamburg, Germany). Fingerprinting by (GTG) 5 PCR included denaturation at $95^{\circ} \mathrm{C}$ for $2 \mathrm{~min}$, followed by $35 \mathrm{cy}$ cles of $94{ }^{\circ} \mathrm{C}$ for $3 \mathrm{~min}, 92^{\circ} \mathrm{C}$ for $30 \mathrm{~s}, 40^{\circ} \mathrm{C}$ for $1 \mathrm{~min}$ and $65^{\circ} \mathrm{C}$ for $8 \mathrm{~min}$, with a final extension of $65^{\circ} \mathrm{C}$ for $8 \mathrm{~min}$. The amplification products were visualized in $1.2 \%$ agarose gels (Genei), which was stained with ethidium bromide. The dendrograms were analyzed using the unweighted pair group method with mathematic averages (UPGMA) and the Dice coefficient cluster analysis with the UVI bandmap software in a gel documentation system (UVI Tec, Cambridge, UK).

Table 1 - Details of the shrimp (Penaeus monodon) -farm-associated bacterial strains in the experiment.

\begin{tabular}{llll}
\hline$V$. harveyi & Source & V. alginolyticus & Source \\
\hline DS134 & Uninfected shrimp intestine & DS29 & Uninfected shrimp intestine \\
DS149 & Water from WSSV infected pond & DS199 & Soil from black spot infected pond \\
DS158 & Soil from WSSV infected pond & DS200 & Soil from reservoir pond of the black spot infected farm \\
DS165 & Uninfected shrimp hepatopancreas & DS246 & Soil from inlet area \\
DS184 & Water with luminescence & DS263 & Soil from Zoothamnium sp. infected pond \\
DS218 & Lesions of black spot infected shrimp & DS334 & Water from WSSV affected pond \\
DS260 & Soil from Zoothamnium sp. infected pond & DS350 & Loose shell affected shrimp intestine \\
\hline
\end{tabular}




\section{Results}

The details of different isolates that were obtained from various sources are provided in Table 1. The isolates that were identified as $V$. alginolyticus were Gram-negative, motile fermentative rods. These rods produced enzymes (catalase, oxidase, gelatinase, lysine and ornithine decarboxylase), acid from sucrose, indole from tryptophan, acetoin from glucose and swarming colonies on agar plates. These isolates showed no growth in the medium with $0 \%$ $\mathrm{NaCl}$ but grew well at $8 \% \mathrm{NaCl}$, which corresponds to the description of $V$. alginolyticus (Alsina and Blanch 1994a, b). However, the $V$. harveyi isolates were different because they were luminescent; they produced no acetoin from glucose and appeared as green or yellow non-swarming colonies in the TCBS agar plates.

The $V$. alginolyticus and $V$. harveyi isolates were further identified by the characteristic amplification of the $16 \mathrm{~S}$ rDNA, and $V$. harveyi was positive for the gyrB gene. The isolates were fingerprinted using IS and GTG PCR, which generated characteristic fingerprint patterns for both species before the experiment at 0 passage. Although the seven $V$. alginolyticus could be differentiated into 4 genogroups in GTG PCR, the seven $V$. harveyi strains formed 3 genogroups during the GTG PCR fingerprint analysis (Figure 1a and $1 \mathrm{~b})$.

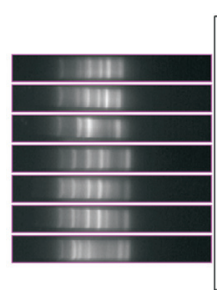

a
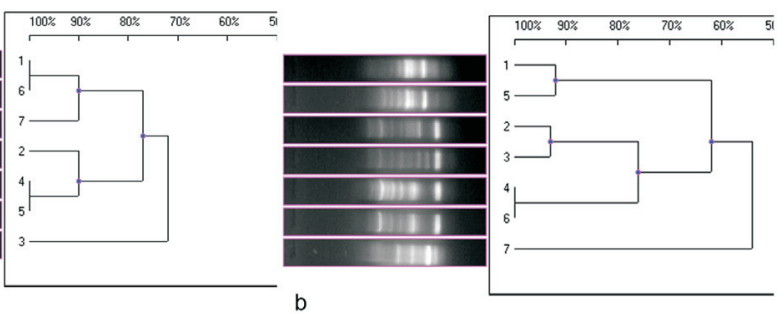

Figure 1 - Fingerprint patterns generated using GTG PCR amplification of the genomic DNA in $1.2 \%$ agarose gel (a) $V$. harveyi strains at 0 passage; lane 1- DS134, lane 2- DS149, lane 3- DS158, lane 4- DS165, lane 5- DS184, lane 6- DS218, lane 7- DS260. (b) V. alginolyticus strains at 0 passage. Lane1DS29, lane 2- DS199, lane 3- DS200, lane 4-DS246, lane 5- DS263, lane 6- DS334, lane 7- DS350.
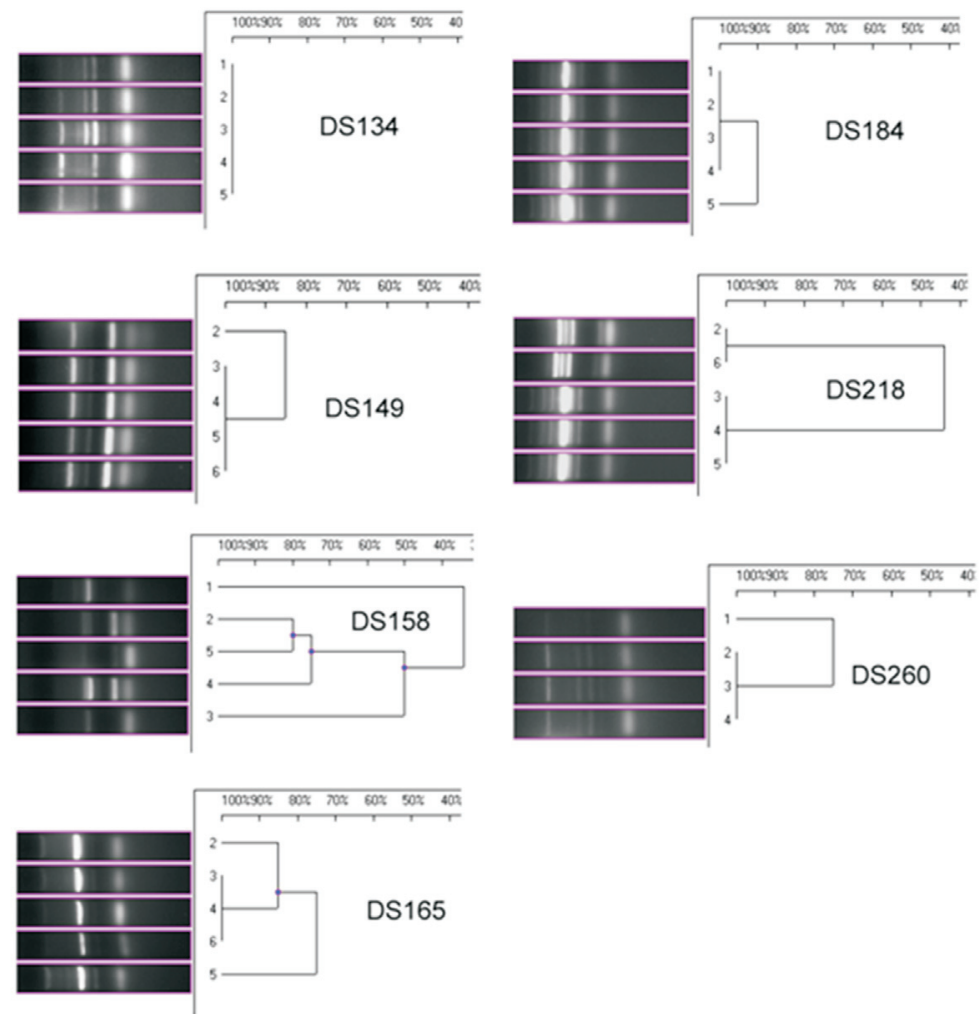

Figure 2 - Fingerprint patterns generated using IS PCR amplification of the genomic DNA of $V$. harveyi with primers, which were targeted at insertion sequences in $1.2 \%$ agarose gel. The number of strains is indicated in each gel. Lane $1-0$ passage, lane 2 to 4 - after 10 passages in 3 , 6 and $8 \%$ NaCl containing medium, respectively, lane 5- after 10 passages in TSA for strains DS134, DS158, DS184 and DS260. Lane 2- 0 passage, lane 3 to 5 - after 10 passages in 3, 6 and $8 \% \mathrm{NaCl}$ containing medium, respectively, lane 6- after 10 passages in TSA for strains DS149, DS165 and DS218. 
Following 10 passages in different salt concentrations, among the $V$. harveyi strains, three strains (DS 134, DS 184 and DS 149) showed no change in the fingerprint pattern (more than $80 \%$ homology) according to the IS PCR analysis (Figure 2). Two isolates DS218 and DS260 exhibited similar types of fingerprint grouping under both IS and GTG PCR analyses (Figures 2 and 3). Among the $V$. alginolyticus strains, the IS and GTG PCR analyses showed a divergence of fingerprint patterns (at less than $80 \%$ homology) for all isolates except DS199, which had a single type of fingerprint pattern (Figures 4 and 5).

\section{Discussion}

The vibrio species continues to be a serious pathogen in shrimp-farming systems, including hatcheries. Because of the ubiquity of the species in the marine and brackish water environments found in the tropical belt, population control is the best management practice in coastal aquaculture systems compared with attempting to avoid the pathogen. Furthermore, many vibrio species are beneficial to the cultured shrimps, whereas others act as opportunistic pathogens. There are instances of potentiation of these opportunistic pathogens, which cause mass mortalities of shrimp
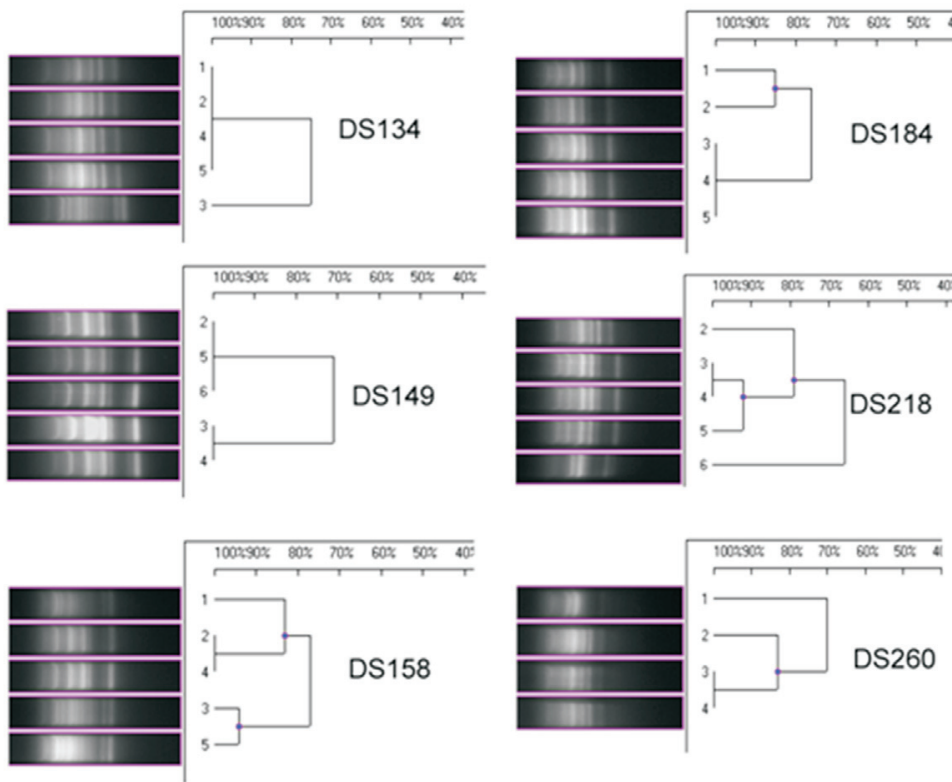
GTG PCR. juveniles and adults. Because these vibrios can act as nitrifiers, they often compete with other heterotrophic bacteria in nitrogen-rich environment and increase the population size, which causes the production of quorum sensing signals and secretion of toxins and biofilms. Although the exact trigger to this conversion is not clear, several factors have been implicated in the generation of virulent strains of vibrios in tropical aquaculture systems (Thompson et al., 2004). Because it has been reported that environmental stresses such as starvation and changes in osmolarity can alter the phenotypic characteristics in $V$. harveyi and $V$. alginolyticus (Xu et al., 2004, 2005; Abdallah et al., 2009), the current study was performed to investigate whether the changed environmental stress could lead to any genomic change in the two vibrio species, which could be detected using fingerprinting PCR techniques such as IS PCR and

The study demonstrated complex genotypic variations that occur in Vibrio strains of tropical aquatic environment because of varied environmental conditions; these genotypic variations result in genetic divergence and/or probable convergence, which are necessitated by adaptive requirements for a successful survival strategy. Previous
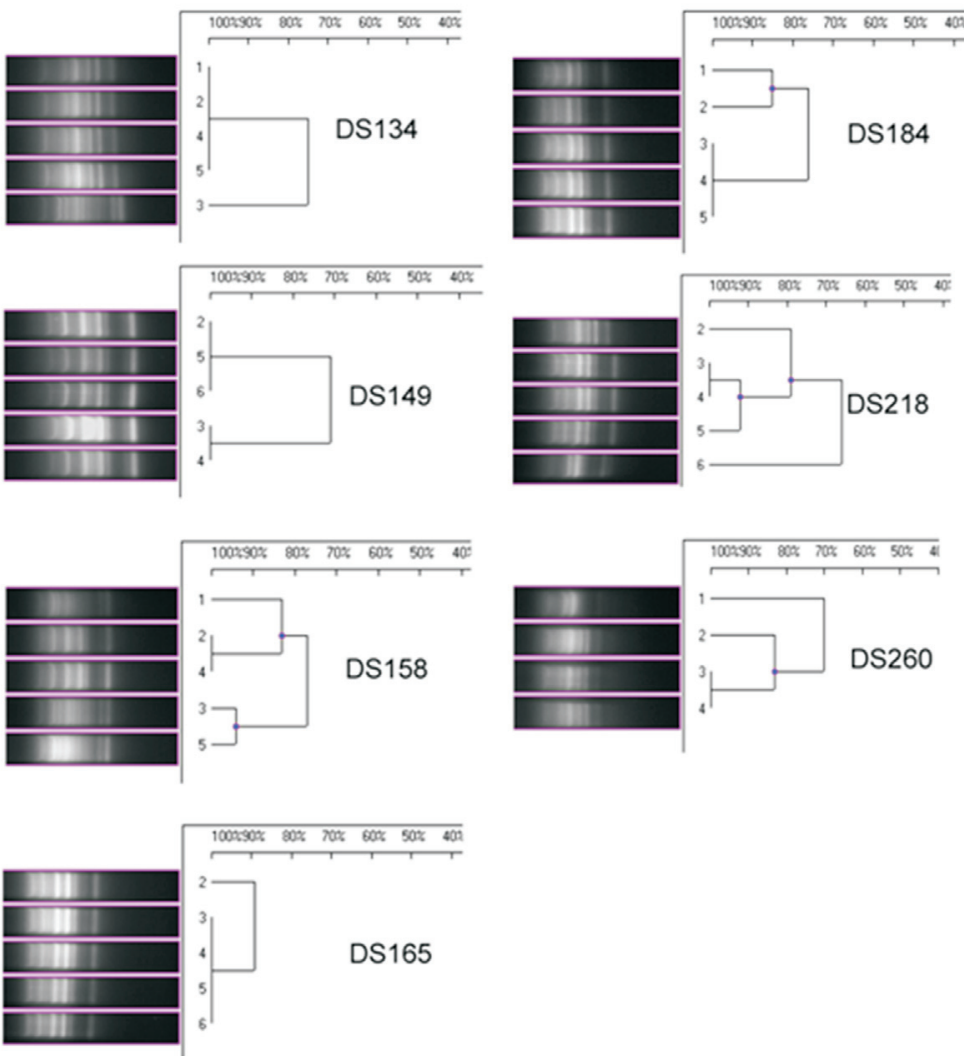

Figure 3 - Fingerprint patterns generated using GTG (5) PCR amplification of the genomic DNA of $V$. harveyi in $1.2 \%$ agarose gel. The number of strains is indicated in each gel. Lane 1- 0 passage, lane 2 to 4 - after 10 passages in 3, 6 and $8 \% \mathrm{NaCl}$ containing medium, respectively, lane 5 - after 10 passages in TSA for strains DS134, DS158, DS184 and DS260. Lane 2- 0 passage, lane 3 to 5- after 10 passages in 3, 6 and 8\% NaCl containing medium, respectively, lane 6- after 10 passages in TSA for strains DS149, DS165 and DS218. 


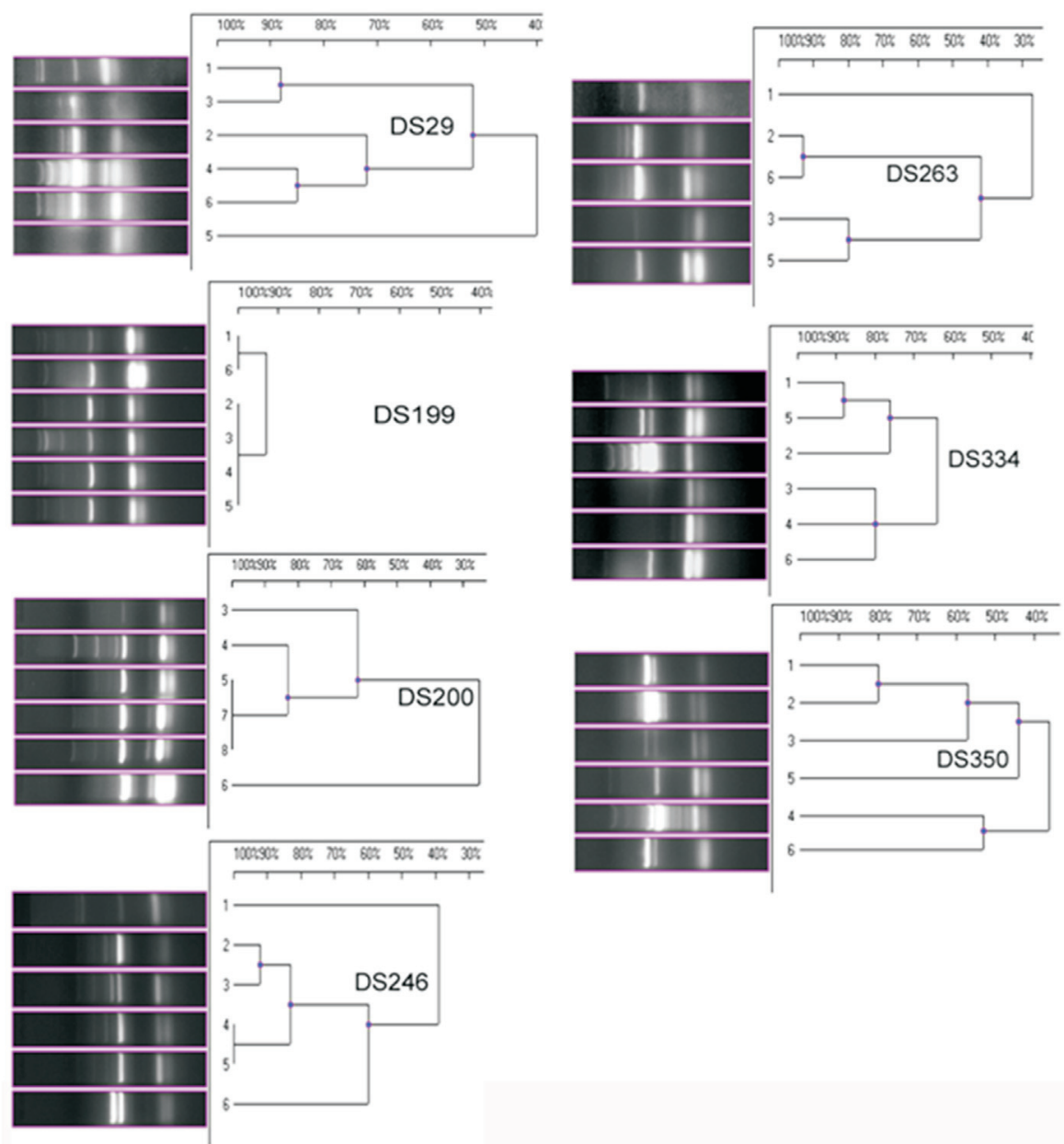

Figure 4 - Fingerprint patterns generated using IS PCR amplification of the genomic DNA of $V$. alginolyticus with primers, which were targeted at the insertion sequences in $1.2 \%$ agarose gel. The number of strains is indicated in each gel. Lane 1 - 0 passage, lane 2 to 5 - after 10 passages in 3,6,8 and $12 \%$ $\mathrm{NaCl}$ containing medium, respectively, lane 6- after 10 passages in TSA for strains DS29, DS199, DS246, DS263, DS334 and DS350. For strain 200, the order starts from 3-8, respectively.

reports have indicated that the environmental and clinical $V$. cholerae strains have similar genomic organization and that pathogenic strains may arise from nontoxigenic strains in the aquatic environment (Chakraborty et al., 2000; Brazil et al., 2002) via multiple horizontal gene transfers (Heidelberg et al., 2000).

The results of the current study indicate that the insertion sequences are notably suitable to determine the strain variation (Chandler, 1998) and play a remarkably important role in the genomic alterations because of their translocating property and functional ability to affect mutation/genetic variation (Syvanen, 1998). Source-independent genogrouping of both $V$. harveyi and $V$. alginolyticus strains was discernable in the current study. Among $V$. harveyi, two strains DS149 and DS158, which originated from WSSV-infected pond, were differentiated into two genogroups. Similarly, two strains that originated from uninfected shrimp intestine (DS134) and isolated from black-spot-infected shrimp lesion (DS218) from two different locations belonged to a single genogroup, with $100 \%$ homogeneity in GTG PCR fingerprinting. Among the $V$. alginolyticus strains, DS246 and DS334 belonged to a single genogroup, although they originated from WSSVinfected pond water and inlet area soil. Similarly, the strains from two distinctly different sources, such as uninfected shrimp intestine (DS29) and soil from Zoothamnium sp.-infected pond (DS263), were grouped into a single genogroup. The current study found that the $V$. harveyi and $V$. alginolyticus strains experienced stress-induced genomic alterations regardless of their source of origin. Changed environmental conditions such as starvation have been reported to cause adaptive mutation involving IS movement (Hall, 1988). The changes noted in the present study, which involved possible stress conditions induced by long-term passaging in high-salt $(\mathrm{NaCl})$ medium and laboratory conditions, would have generated the mutation involving IS elements. A similar situation might also occur for the organisms that were growing in vivo, where the bacterial isolates would experience a changed propagating en- 


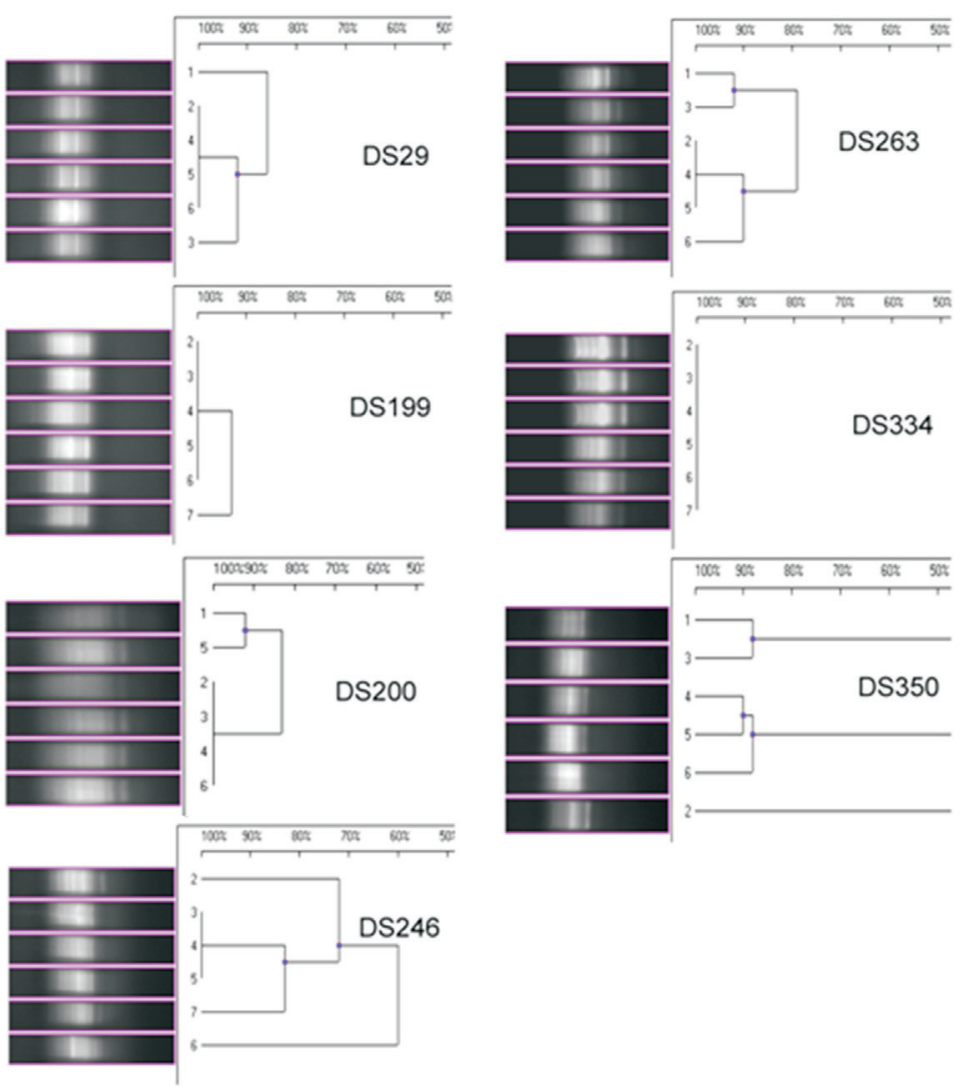

Figure 5 - Fingerprint patterns generated using GTG (5) PCR amplification of the genomic DNA of $V$. alginolyticus in $1.2 \%$ agarose gel. The number of strains is indicated in each gel. Lane 1- 0 passage, lane 2 to 5 - after 10 passages in 3, 6, 8 and $12 \% \mathrm{NaCl}$ containing medium, respectively, lane 6- after 10 passages in TSA for strains DS29, DS200, DS263 and DS350. Lane 2- 0 passage, lane 3 to 6- after 10 passages in 3, 6, 8 and $12 \% \mathrm{NaCl}$ containing medium, respectively, lane 7- after 10 passages in TSA for strains DS199, DS246 and DS334.

vironment, which altered the fingerprint pattern from the original isolates. Similarly, such a change might occur in the isolates from animals that experienced a few cycles of passaging through the laboratory medium before they were fingerprinted using IS PCR. The $V$. vulnificus strains were reported to convert between distinct phenotypes of encapsulated opaque and nonencapsulated translucent forms; in addition, they switched from these variants to a rugose form under environmentally challenging conditions of low temperature (Grau et al., 2005). The rugose variant, which was produced at temperatures below $37^{\circ} \mathrm{C}$ and could form prodigious biofilms, has been found to have an important role in aiding the survival of the species at cooler temperatures in its natural marine environment, in nutritionally deficient conditions or otherwise unfavorable conditions. Nevertheless, the rugose variant potentiates the pathogenicity characteristics within the human host. The present study shows the genotypic changes that can occur in $V$. harveyi and $V$. alginolyticus strains that are subjected to altered propagating conditions. The role of IS sequences in revealing such changes has been reported in cases of enteric bacteria such as E. coli (Syvanen, 1998).

Our results demonstrate the genetic instability of two important and cosmopolitan Vibrio species of shrimp- farming systems. In a similar study, where $V$. alginolyticus and $V$. parahaemolyticus were maintained in low nutrient conditions, Abdallah et al. (2009) found that the adaptive response speed of vibrios to starvation was variable, and $V$. alginolyticus more quickly modified its extra chromosomal genetic content compared with $V$. parahaemolyticus to adapt to the changed environmental conditions. In the present study, changes were also visible in the genomic characteristics of $V$. alginolyticus and $V$. harveyi when they were fingerprinted using GTG (5) and IS PCR techniques. These techniques show that the genomic plasticity of the vibrios in the environment is probably a survival strategy that can also result in the phenotypic alterations in the protein expression (Xu et al., 2004, 2005).

The findings of the present study is significant in the current pandemic scenario in the shrimp-farming sector, where the Early Mortality Syndrome (EMS) or Acute Hepatopancreatic Necrosis Disease (AHPND) significantly decreases shrimp production worldwide, which leads to huge economic losses to many countries. AHPND has been reported to be caused by a specific strain of $V$. parahaemolyticus (Tran et al., 2013), which was not hith- 
erto reported from shrimp aquaculture within the last two decades.

\section{Acknowledgments}

Financial assistance from the Department of Science and Technology, Govt. of India, New Delhi to MRG is gratefully acknowledged. The work formed part of the M.F.Sc thesis of the first author, which was submitted to the Tamilnadu Veterinary and Animal Sciences University.

\section{References}

Abdallah FB, Kallel H, Bakhrouf A (2009) Enzymatic, outer membrane proteins and plasmid alterations of starved Vibrio parahaemolyticus and Vibrio alginolyticus cells in seawater. Mol Microbiol 191:493-500.

Alsina M, Blanch AR (1994a) A set of keys for the biochemical identification of environmental vibrios. J Appl Bacteriol 76:79-85.

Alsina M, Blanch AR (1994b) Improvement and update of a set of keys for biochemical identification of Vibrio species. J Appl Bacteriol 77:719-721.

Brazil JM, Alves RM, Rivera ING et al. (2002) Prevalence of virulence-associated genes in clinical and environmental Vibrio cholerae strains isolated in Brazil between 1991 and 1999. FEMS Microbiol Lett 215:15-21.

Cano-Gomez A, Bourne DG, Hall MR et al. (2009) Molecular identification, typing and tracking of Vibrio harveyi in aquaculture systems: current methods and future prospects. Aquaculture 287:1-10.

Chakraborty S, Mukhopadhyay AK, Bhadra RK et al. (2000) Virulence genes in environmental strains of Vibrio cholerae. Appl Environ Microbiol 66:4022-4028.

Chandler MS (1998) Insertion sequences and transposons. In: De Bruijn FJ, Lupski JR, Weinstock GM (eds). Bacterial Genomes. International Thomson Publishing, Florence, p. 30-36.

Defoirdt T, Verstraete W, Bossier P (2007) Luminescence, virulence and quorum sensing signal production by pathogenic Vibrio campbellii and Vibrio harveyi isolates. J Appl Microbiol 104:1480-1487.

George MR, John KR, Iyappan T et al. (2005) Genetic heterogeneity among Vibrio alginolyticus isolated from shrimp farms by PCR fingerprinting. Lett Appl Microbiol 40:369-372.

Gomez-Gil B, Soto-Rodriguez AS, Garcia-Gasca A et al. (2004) Molecular identification of Vibrio harveyi related isolates associated with diseased aquatic organisms, Microbiol 150:1769-1777.

Grau BL, Henk MC, Pettis GS (2005) High-frequency phase variation of Vibrio vulnificus 1003: Isolation and characterization of a rugose phenotypic variant. J Bacteriol 187:2519-2525.

Grimes DJ, Singleton FL, Colwell RR (1984) Allogenic succession of marine bacterial communities in response to pharmaceutical waste. J Appl Bacteriol 57:247-261
Hall BG (1988) Adaptive evolution that requires multiple spontaneous mutations. I. Mutations involving an insertion sequences. Genetics120:887-897.

Heidelberg JF, Eisen JA, Nelson WC et al. (2000) DNA sequence of both chromosomes of the cholera pathogen Vibrio cholerae. Nature 406:477-483.

Hernandez G, Olmos J (2004) Molecular identification of pathogenic and nonpathogenic strains of Vibrio harveyi using PCR and RAPD. Appl Microbiol Biotechnol 63:722-727.

Lavilla-Pitogo CR, Baticados MCL, Cruz-Lacierda ER et al. (1990) Occurrence of luminous bacterial disease of Penaeus monodon larvae in the Philippines. Aquaculture 91:1-13.

Lavilla-Pitogo CR, de la Pena LD (1998) Mortalities of pond cultured juvenile shrimp, Penaeus monodon, associated with dominance of luminescent vibrios in the rearing environment. Aquaculture 164:337-349.

Leano EM, Lavilla-Pitogo CR, Paner MG (1998) Bacterial flora in the hepatopancreas of pond-reared Penaeus monodon juveniles with luminous vibriosis. Aquaculture 164:367-374.

Lo CF, Leu JH, Ho CH et al. (1996) Detection of baculovirus associated with white spot syndrome (WSBV) in penaeid shrimps using polymerase chain reaction. Dis Aquat Org 25:133-141.

Manefield M, Harris L, Rice SA et al. (2000) Inhibition of luminescence and virulence in the black tiger prawn $(P$. monodon) pathogen Vibrio harveyi by intercellular signal antagonists. Appl Environ Microbiol 66:2079-2084.

Oakey HJ, Levy N, Bourne DG et al. (2003) The use of PCR to aid in the rapid identification of Vibrio harveyi isolates. J Appl Microbiol 95:1293-1303.

Ruwandeepika HAD, Jayaweera TSP, Bhowmick PP et al. (2012) Pathogenesis, virulence factors and virulence regulation of vibrios belonging to the Harveyi clade. Rev Aquaculture 4:59-74.

Sambrook J, Fritsch EF, Maniatis T (1989) Molecular cloning: a laboratory manual. $2^{\text {nd }}$ ed. Cold Spring Harbor Laboratory Press, New York.

Satendrakumar, George MR, John KR et al. (2007) Molecular typing of bacteria Vibrio harveyi and $V$. alginolyticus from shrimp farming system. Ind J Mar Sci 36:43-50.

Sawabe T, Kita TK, Thompson FL (2007) Inferring the evolutionary history of vibrios by means of multilocus sequence analysis. J Bacteriol 189:7932-7936.

Syvanen M (1998) Insertion sequences and their evolutionary role. In: De Bruijn FJ, Lupski JR, Weinstock GM (eds). Bacterial Genomes. International Thomson Publishing, Florence, 213-218.

Thaithongnum S, Ratanama P, Weeradechapol K et al. (2006) Detection of $V$. harveyi in shrimp postlarvae and hatchery tank water by the most probable number technique with PCR. Aquaculture 261:1-9.

Thangarani JA (2001) Role of beneficial bacteria in water quality and pathogen control in shrimp ponds 125 p. (M.F.Sc. Thesis. Department of Aquaculture, Fisheries college and Research Institute, Tamil Nadu Veterinary and Animal Sciences University, Thoothukudi, India).

Thompson FL, Iida T, Swings J (2004) Biodiversity of vibrios. Microbiol Mol Biol Rev 68:403-431. 
Tran L, Nunan L, Redman RM et al. (2013) Determination of the infectious nature of the agent of acute hepatopancreatic necrosis syndrome affecting penaeid shrimp. Dis Aquat Org 105:45-55.

Urakawa H, Rivera ING (2006) Aquatic environment. In: Thompson, F.L., Austin, B., Swings, J. (eds). The biology of vibrios. ASM Press, Washington, DC, pp 175-189.
Xu C, Ren H, Wang S et al. (2004) Proteomic analysis of salt sensitive outer membrane proteins of Vibrio parahaemolyticus. Res Microbiol 155:835-842.

Xu C, Wang S, Ren H et al. (2005) Proteomic analysis on the expression of outer membrane proteins of Vibrio alginolyticus at different sodium concentrations. Proteomics 5:31423152 .

Associate Editor: Cynthia Canêdo da Silva

All the content of the journal, except where otherwise noted, is licensed under a Creative Commons License CC BY-NC. 